\title{
The Evaluation of Typical Connection Mode of Distribution Network Based on TOPSIS
}

\author{
Bingqian Song \\ North China Electric Power University, Beijing, 102206 \\ alanncepu@foxmail.com
}

Keywords: Comprehensive evaluation system; TOPSIS; Entropy weight method

\begin{abstract}
The distribution network plays a very important role on the transmission network and the power users, is responsible for ensuring reliable and continuous power supply and providing a good power quality. This paper aims to establish a mathematical model based on TOPSIS with integrated reliability, flexibility and economic indicators to assess the three aspects of distribution network connection mode, and entropy weight method is used to determine the proportion of each index in the evaluation analysis. To a certain extent, it avoids the subjectivity and randomness of the traditional TOPSIS method, and it can be more operational and more objective. It can reflect the implicit information of the data, the difference of the index and the significance of the resolution. Finally, this article analyzes and judges through the actual example the pros and cons of the connection mode.
\end{abstract}

\section{Introduction}

The evaluation of distribution network generally refers to the evaluation of the reliability, flexibility and economy of the connection mode of distribution network according to certain boundary conditions. At present, the comprehensive evaluation method used at home and abroad can be divided into two categories according to the method of determining weights. One kind is the subjective weighting method, taking the comprehensive consultation grade to determine the weight of each factor, such as the comprehensive index method, fuzzy comprehensive evaluation method, the efficiency coefficient method, etc., This kind of method is more subjective in the evaluation and analysis, which may be due to the evaluation of their own reasons to make the evaluation results appear deviation. Another kind of weight is more objective, according to the correlation between each evaluation index or the variation degree of each evaluation index value to determine its weight, such as principal component analysis, factor analysis, TOPSIS method and so on. According to the theory of structure element, the paper has made the connection mode of medium voltage distribution network based on [2]; A comprehensive decision making method of high voltage distribution network connection mode based on interval analytic hierarchy process is proposed in the paper[3]; The paper proposed a method of determining the weights by analytic hierarchy process, and applied to the evaluation of the investment scheme of distribution network planning by using the multiple criteria decision making method of the general weighting and the method of calculating the value of the [4].

The purpose of this paper is to use an objective evaluation method for the comprehensive evaluation of distribution network connection mode, which can make full use of the information in the alternative scheme, to combine of qualitative and quantitative analysis provides a new way for the evaluation and selection of distribution network connection mode.

\section{Connection Mode and Evaluation Method}

Typical Connection Mode of Distribution Network and Its Evaluation Index. City distribution network composed of overhead lines and cable mixed. In the study of the network structure of the $10 \mathrm{kV}$ distribution network in a certain power supply area, we analyze the overhead line and the cable line separately. In this paper, through the analysis, it summarizes the current distribution network typical wiring classification, such as Fig. 1. 


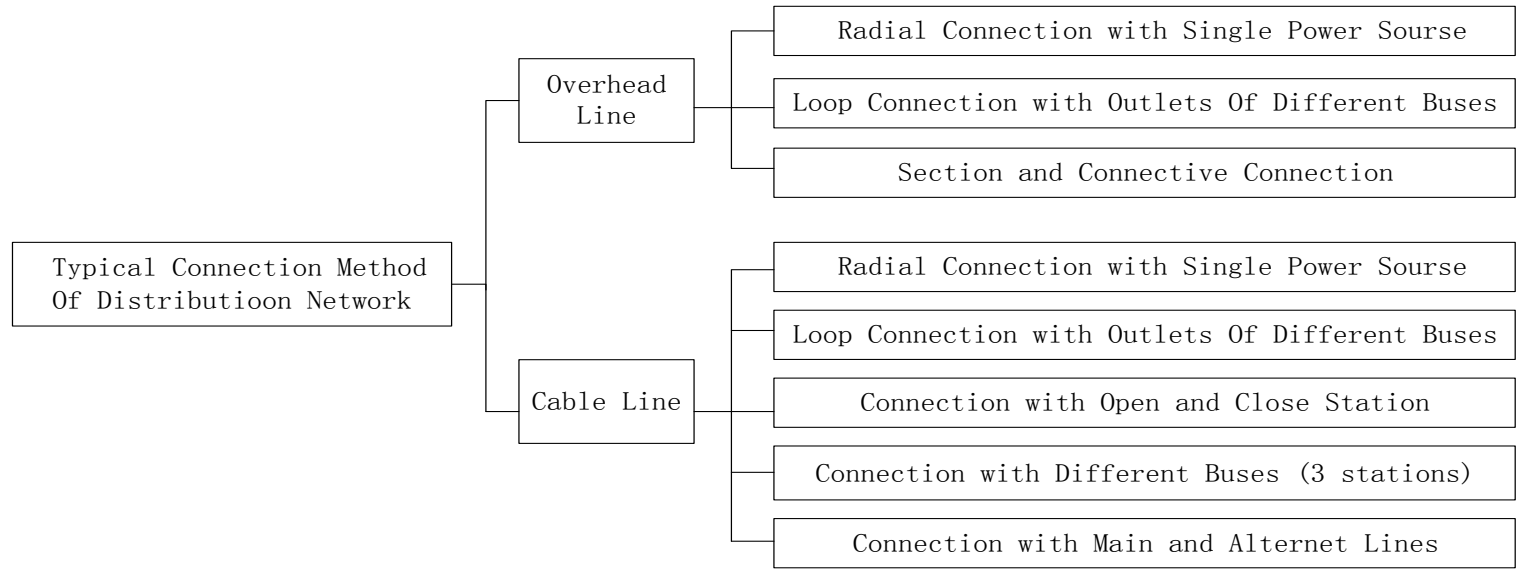

Figure 1. Typical connection modes of distribution network

Based on the classification of the connection mode of distribution network, the three typical evaluation indexes of the reliability, flexibility and economy are selected to evaluate the distribution network.

Reliability evaluation index adopts 3 indexes: system average interruption frequency index (SAIFI), system average interruption duration index (SAIDI) and average power supply reliability index (ASAI).[2]

Reserve capacity is used as an important index to evaluate the flexibility of connection mode in power system.

Table 1 Spare capacity in different connection modes

\begin{tabular}{|c|c|}
\hline Connection mode & Spare capacity \\
\hline Radial Connection with Single Power Source & $1 / 2$ \\
\hline Loop Connection with Outlets Of Different Buses & $1 /(\mathrm{n}+1)$ \\
\hline Section and Connective Connection & $1 / 2$ \\
\hline Connection with Open and Close Station & $1 / 3$ \\
\hline Connection with Different Buses (3 stations) & $1 / 3$ \\
\hline Connection with Main and AlterNet Lines & 0 \\
\hline
\end{tabular}

Economic evaluation mainly includes two aspects: financial benefit evaluation and technical and economic benefit evaluation. The greater the financial net present value rate NPVR and the internal rate of return IRR is, the better economic benefits of the program is. In the evaluation of technical and economic benefits, the maximum expected return of the total assets of the unit is used as the evaluation index FP. The calculation formula can be referenced in the literature [2]. The index reflects the contribution of the unit total assets for the power grid, in addition to considering the ability of power supply, also considering the reliability, power loss, tariffs and other factors. The index value is greater, the grid technology and economy is better.

TOPSIS Method and Its Application. Using Ci express the extent of the standard wiring way, $\mathrm{C}_{\mathrm{i} 1}$ showing the reliability after the standardization of the connection mode, $\mathrm{C}_{\mathrm{i} 2}$ shoeing the flexibility after the standardization of the wiring, $\mathrm{C}_{\mathrm{i} 3}$ showing the connection mode of economy after the standardization. Comparing the alternatives of the solution set $\mathrm{C}$ with the distance of the positive ideal solution and the negative ideal solution, the best solution of the solution set $\mathrm{C}$ is not only close to the positive ideal solution but also far away from the negative ideal solution. The 
specific steps of the evaluation and analysis of the connection mode by using the TOPSIS method are as follows

(1) Data standardization. The decision matrix of original connection mode is $A=\left(a_{i j}\right)_{m \times n}$, after the transformation, the decision matrix denoted as $C=\left(c_{i j}\right)_{m \times n}$. The maximum value in the column $j$ of the decision matrix is $a_{j}^{\max }$ and the minimum value in the column $j$ of the decision matrix is $a_{j}^{\min }$. Due to the reliability, flexibility, and economic attribute value for benefit type, that is, the bigger as the better, the standard decision matrix is:

$$
c_{i j}=a_{i j} / a_{j}^{\max }
$$

(2)Entropy weight method determines the weight of the index, so as to construct the weighted decision matrix. The calculation steps are:

$$
\begin{gathered}
H_{j}=-\frac{1}{\ln m} \sum_{i=1}^{m} f_{i j} \ln f_{i j} ; f_{i j}=\frac{c_{i j}}{\sum_{i=1}^{m} c_{i j}} \\
\omega_{\mathrm{j}}=\frac{1-H_{j}}{3-\sum_{j=1}^{3} H_{j}}
\end{gathered}
$$

In the formula, 3 indicates that there are 3 indexes of reliability, flexibility and economy, $\mathrm{H}_{\mathrm{j}}$ is the item $\mathrm{j}$ entropy of indicators; $\omega_{\mathrm{j}}$ is the item $\mathrm{j}$ weigh value which is satisfied to $0 \leq \omega_{\mathrm{j}} \leq 1$ and $\omega_{1}+\omega_{2}+\omega_{3}=1$.

Combined weight coefficient, by normalizing each element multiplies the corresponding weight coefficient on decision matrix, getting weighted decision matrix $\mathrm{C}^{\prime}=\left(\mathrm{c}_{\mathrm{ij}}^{\prime}\right)_{\mathrm{m} \times 3}$, where, $c_{\mathrm{ij}}^{\prime}=\omega_{j} c_{i j}\left({ }^{\prime}\right.$ is bypassed as follows).

(3)Determine the positive ideal solution $\mathrm{C}^{*}$ and the negative ideal solutionC $\mathrm{C}^{0}$. The assumes that the first $j(j=1,2,3)$ property value of positive ideal solution is $c_{j}{ }^{*}$, The first $J(j=1,2,3)$ property value of the negative ideal solution is $c_{-} j^{\wedge} 0$, According to the different connection mode of the index value, there are:

$$
\begin{gathered}
c_{j}^{*}=\max _{i} c_{i j}, i=1,2,3 \ldots, m ; j=1,2,3 \\
c_{j}^{0}=\min _{i} c_{i j}, i=1,2,3 \ldots, m ; j=1,2,3
\end{gathered}
$$

(4)Calculating distance. Separately calculate each area of each time interval the distance from the evaluation vector to the negative ideal solution $\mathrm{D}^{*}$ and the positive ideal solution $\mathrm{D}^{0}$.

$$
\begin{aligned}
& D_{i}^{*}=\sqrt{\sum_{j=1}^{3}\left(c_{i j}-c_{j}^{*}\right)^{2}} \quad(j=1,2,3) \\
& D_{i}^{0}=\sqrt{\sum_{j=1}^{3}\left(c_{i j}-c_{j}^{0}\right)^{2}} \quad(j=1,2,3)
\end{aligned}
$$

(5)Calculate the close degree $\mathrm{Ni}$ of the evaluation object and the optimal supply and demand matching target value at each time interval.

$$
N_{i}=\frac{D_{i}^{0}}{D_{i}^{0}+D_{i}^{*}}
$$


Where: The larger the $\mathrm{Ni}$ is, the more close to the optimal level the i connection mode is, close degree $0 \sim 1$ value between $\mathrm{Ni}$.

\section{Case Analysis}

In order to evaluate the different connection modes in the different area, data processing is carried out according to the entropy weight TOPSIS method proposed in section second. In order to simplify the calculation, it assumed that there are 1 regional planning of $110 \mathrm{kV}$ substation, the capacity is $\mathrm{P}=3 * 50=150 \mathrm{MW}$, the load rate is $=67 \%$. For ease of handling,

In the planning area, a single source radiation, a double power supply with different bus lines and three subsection three are used as the three connection modes, as shown in Fig. 2, 3 and4.

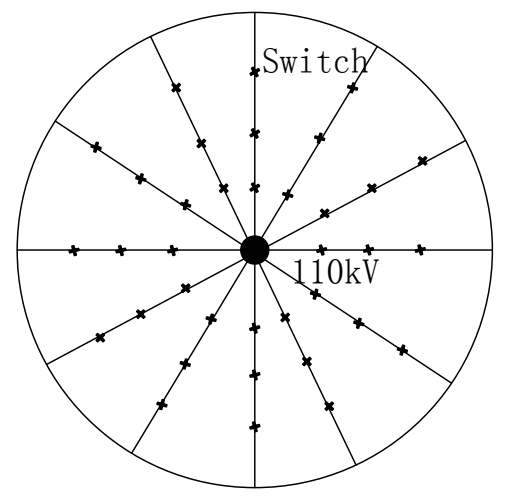

Figure 2. Connection mode 1

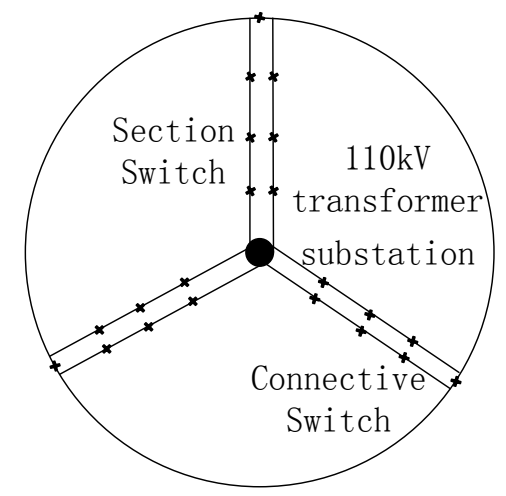

Figure 3. Connection mode 2

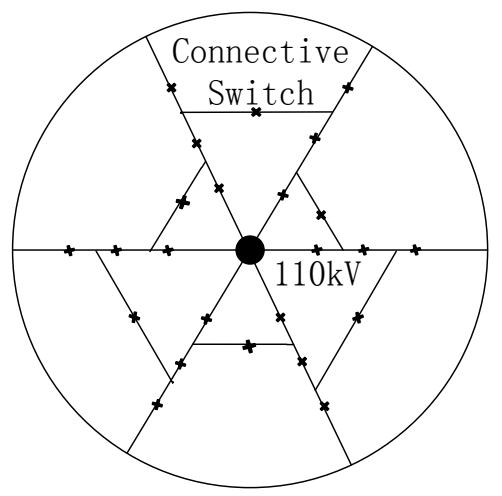

Figure 4. Connection mode 3

First, according to the basic data and the entropy weight method to the corresponding in Section 2 , data of the reliability, flexibility, economic indicators is pretreated. Second, according to the relative value of the three indexes under the three connection modes, in accordance with the second section TOPSIS method to analyze and deal with, then the decision value of the weighted norm is obtained as shown in Table 2.

Table 2 Calculation results of each index

\begin{tabular}{|c|c|c|c|c|c|c|}
\hline \multirow{2}{*}{$\begin{array}{c}\text { Connection } \\
\text { mode }\end{array}$} & \multicolumn{2}{|c|}{ Reliability index } & \multicolumn{2}{c|}{ Flexibility index } & \multicolumn{2}{c|}{ Economic index } \\
\cline { 2 - 7 } & $\begin{array}{c}\text { Normative } \\
\text { decision } \\
\text { value }\end{array}$ & $\begin{array}{c}\text { Weighted } \\
\text { norm } \\
\text { decision } \\
\text { value }\end{array}$ & $\begin{array}{c}\text { Normative } \\
\text { decision } \\
\text { value }\end{array}$ & $\begin{array}{c}\text { Weighted } \\
\text { norm } \\
\text { decision } \\
\text { value }\end{array}$ & $\begin{array}{c}\text { Normative } \\
\text { decision } \\
\text { value }\end{array}$ & $\begin{array}{c}\text { Weighted } \\
\text { norm } \\
\text { decision } \\
\text { value }\end{array}$ \\
\hline 1 & 0.473 & 0.046 & 0 & 0 & 1 & 0.0141 \\
\hline 2 & 0.645 & 0.063 & 1 & 0.887 & 0.802 & 0.0113 \\
\hline 3 & 1 & 0.098 & 0.66 & 0.585 & 0.771 & 0.0109 \\
\hline $\begin{array}{c}\text { Weight } \\
\text { coefficient }\end{array}$ & \multicolumn{2}{|c|}{0.0981} & \multicolumn{2}{c|}{0.8876} & & 0.0141 \\
\hline
\end{tabular}

According to the data in Table 2, the ideal solution is $(0.098168,0.887675,0.014157)$, the negative ideal solution for $(0.046528,0,0.010924)$, the calculation of the three wiring methods of the schedule as shown in Table 3 
Table 3 Calculation results of close degree

\begin{tabular}{|c|c|c|c|}
\hline Connection mode & $\mathrm{D}^{*}$ & $\mathrm{D}^{0}$ & Close degree $\mathrm{N}$ \\
\hline 1 & 0.7906 & 0.00001045 & 0.000013 \\
\hline 2 & 0.0012 & 0.7882 & 0.9984 \\
\hline 3 & 0.0911 & 0.3459 & 0.7915 \\
\hline
\end{tabular}

From the Table 3, 4, in the comprehensive requirements of the high reliability, high flexibility and high economy, wiring 2 shows a good advantage. Because the weight of the selection is based on the change of the index, that is, the impact of changes on the quality of the connection mode of the size, so the results are objective, does not change with the subjective factors of planners.

\section{Conclusion}

The paper though the analysis of traditional evaluation index in chinses connection mode of the existing urban medium voltage distribution network, according to the reliability, flexibility and economy index, a comprehensive evaluation system of distribution network typical connection mode based on entropy weight TOPSIS method is proposed, then the proposed comprehensive evaluation system is used to select the typical connection mode of the planning area. The system entropy method is used to determine index weight to avoid the subjectivity of expert experience method, to a certain degree objectively reflecting the level of the pros and cons of the connection mode, which can provide reference for the choice of distribution network structure and has a certain practical significance.

\section{References}

[1] Billinton R, Billinton J. Distribution system reliability indices [J]. IEEE Transaction on Power Delivery, 1989, 4(1): 561-568.

[2] Liu Xiang jun, MA Shuang \& XU Gang, (2012) "Formation of Typical Connection Mode for Distribution Network by Elementary Connection Model", Power System Technology, Vol.36, No. 2, pp59-63.

[3] Li Jianlin, Zhang Zhongchao. The calculation and analysis of $10 \mathrm{kV}$ power distribution system in Datong area [J]. Proceedings of the CSU-EPSA, 2002, 14(4): 61-63, 75.

[4] Liu Zifa, Guo Huimeng \& Li Mengyu, (2014) "Study on Power Grid Blackout risk under Different Connection Modes”, Electric power construction, Vol. 35, No.8, pp25-29.

[5] Liu Gui-Long, (2004) "The Axiomatic Systems of Rough Fuzzy Sets on Fuzzy Approximation Spaces”, CHINESE JOURNAL OF COMPUTERS, Vol. 27, No.9, pp1188-1191.

[6] Sun Lifeng. Research on connection mode of $10 \mathrm{kV}$ distribution network in city power network layout [J]. Guangdong Science and Engineering, 2009, 25(12): 23-25(in Chinese).

[7] Wang Cheng-shan, Wang Sai-yi, GE Shao-yun, et al. Economy and reliability analysis of different connection modes in MV distribution networks [J]. Automation of Electric Power Systems, 2002, 26(24):34-39.

[8] Ling Yong-biao. Study on electric connection mode of distribution network to match up to distribution automation development [J]. Modern Electric Power, 2005, 22(1):45-47.

[9] [Guo Yong-ji. Reliability evaluation of distribution system with consideration of capacity constraint [J]. Automation of Electric Power Systems, 2000, 24(17):48-52.

[10]Wan Guocheng, Ren Zhen, Tian Xiang. Study on model of reliability network equivalent of distribution system reliability evaluation [J]. Proceedings of the CSEE, 2003, 23(5): 48-52. 